\title{
Nerve involvement in fluid transport in the inflamed rat jejunum
}

\author{
$M$ Jodal, $\mathrm{U}$ Wingren, $M$ Jansson, $M$ Heidemann, $O$ Lundgren
}

\begin{abstract}
Net fluid transport was measured in denervated jejunal segments of rats infected with larvae of Nippostrongylus brasiliensis. On days 6-9 after nematode inoculation, when the jejunal segment exhibited macroscopic and microscopic signs of inflammation, net fluid absorption was noticeably attenuated compared with control, and in eight of 26 experiments a net fluid secretion was seen. To determine whether enteric nerves participated in the response, intravenous hexamethonium $(10 \mathrm{mg} /$ $\mathrm{kg}$ body weight) was given or lidocaine (1\% solution) was placed on the serosa of the intestinal segment. Both drugs significantly reduced fluid secretion or increased fluid absorption. The effect was more pronounced the lower the rate of fluid absorption or the higher the rate of fluid secretion. The inflammatory response influenced intestinal fluid transport partly via activation of the enteric nervous system. It was estimated that $50-60 \%$ of the change in fluid transport caused by the parasite could be ascribed to activation of intramural nervous reflexes. The effect of hexamethonium indicates that a cholinergic synapse is present in the secretory nervous reflux activated by inflammation. Experiments were also performed on animals on days 11-14 after infection when the nematodes had been expelled from the animal. A large net fluid absorption was then recorded.

(Gut 1993; 34: 1526-1530)
\end{abstract}

During the past decade evidence has been accumulating that fluid secretion in the small intestine is often evoked via stimulation of the enteric nervous system (ENS). This was originally suggested by the work of Cassuto et al, who, in a series of reports, showed that secretion evoked by cholera toxin in anaesthetised rats and cats was mediated to a large extent via the ENS. ${ }^{1-4}$ It was subsequently shown that this was also the case for intestinal secretion caused by the heat stable enterotoxin from Escherichia coli, certain bile salts, ricinoleic acid, serotonin, and prostaglandin $\mathrm{E}_{2}$ (for reviews consult Lundgren et al $^{5}$ and Jodal ${ }^{6}$ ).

The role of the ENS in the secretory response to 5-hydroxytryptamine and prostaglandins sug gested that nerves may also be involved in secretory states accompanying an inflammatory response. This proposal was tested in rat experiments by Brunsson, ${ }^{7}$ who showed that the fluid secretion evoked by an invasive strain of Salmonella typhimurium (R5) was turned into absorption after giving intravenous hexamethonium or by exposing the intestinal serosa to lidocaine, a local anaesthetic.
This study was designed to investigate further the relationship between inflammation and fluid transport in the small intestine of the rat. For this purpose rats were inoculated with Nippostrongylus brasiliensis by injecting the parasites subcutaneously. On day 3 the nematodes reach the small intestine and on days 6-8 an inflammatory response in the jejunum is seen. Ten to 12 days after inoculation, the worms are expelled from the small intestine and the inflammatory response fades away. Fluid transport was measured in vivo in rats before and after expulsion of the parasite and the possible involvement of ENS in the response was tested pharmacologically.

\section{Methods}

ANIMALS

Sprague-Dawley rats (Alab AB, Stockholm, Sweden) weighing $300-400 \mathrm{~g}$ were given a subcutaneous injection of 6000 larvae of Nippostrongylus brasiliensis in $0.5 \mathrm{ml}$ saline. Rats were housed in standardised environmental conditions $\left(22^{\circ} \mathrm{C}, 60 \%\right.$ humidity, light $6 \cdot 00-18.00$ hours). The animals were studied on days 6-9 or 11-14 after being injected with larvae.

\section{ANESTHESIA AND OPERATIVE PROCEDURES}

Anaesthesia was induced by mebumal (60 mg. $\mathrm{kg}^{-1}$ body weight) and maintained with chloralose (100 mg. $\mathrm{kg}^{-1} \mathrm{bw}$ ) as a bolus plus $20 \mathrm{mg} \cdot \mathrm{h}^{-1} \cdot \mathrm{kg}^{-1}$ body weight as an infusion. A tracheotomy was performed and a tracheal cannula was inserted to maintain a free airway. Catheters were inserted into the femoral artery and vein for blood pressure recordings and drug administration, respectively. To prevent acidosis due to the operative trauma an infusion of a glucose-bicarbonate solution (see below) was given intra-arterially at a rate of $0.03 \mathrm{ml} . \mathrm{min}^{-1}$. A Statham pressure transducer (Model P23AC) was connected to the arterial catheter and arterial pressure was continuously recorded on a Grass polygraph.

The abdomen was opened by midline incision. A 5 to $10 \mathrm{~cm}$ long segment of the jejunum was selected. In the experiments performed on days 6-9 after injecting the larvae, the part of the jejunum with the most severe signs of inflammation was selected for the experiment. The remainder of the small intestine and the colon were removed. All nerves in the tissues around the superior mesenteric artery were cut, thereby accomplishing denervation of the extrinsic autonomic nervous supply to the intestinal segment.

The rat was placed on a thermistor controlled operating table. The rat, except for its head and 
the opening of the tracheal cannula, was then placed in a plastic box. In the box the humidity was high and the temperature was maintained at $35^{\circ} \mathrm{C}$ by a lamp outside and a thermocouple sensor inside. The temperature of the animal was monitored continuously by a thermocouple thermometer in the mouth. In this way body temperature was kept constant and the evaporation of water from abdominal tissues was minimised.

RECORDING OF INTESTINAL NET FLUID TRANSPORT Net fluid transport was measured in two ways. In some experiments net fluid transport was measured by the method originally described by Cassuto et al. ${ }^{8}$ The intestinal segment, covered by a thin plastic sheet, was placed on a plastic plate specially designed for this purpose. The distal end of the intestinal segment was connected via a plastic tube to a basin on the plate. This allowed free passage of fluid in and out of the segment, confirmed by flushing the segment via a cannula in the proximal end. The plastic plate was suspended $3-5 \mathrm{~mm}$ above the abdominal wall from a force displacement transducer (Grass FT3C).

The other method used for fluid transport measurements was similar to the one just described, the only difference being that the segment was continuously perfused at a rate of $0 \cdot 1$ ml.min ${ }^{-1}$ by a pump (Isamatec, Switzerland) via a closed system connecting the two ends of the segment. The system contained a reservoir (volume $25 \mathrm{ml}$ ) to minimise recirculation (for details, see Jodal $e t a l^{9}$ ).

Net fluid transport was considered to be equal to changes in the intestinal weight, which was continuously monitored by connecting the force transducer to a Grass polygraph. Net fluid transport was calculated from registration periods lasting for at least 15 minutes and related to the serosal surface area measured after the experiment. Net fluid transport was expressed as $\mu \mathrm{l} . \mathrm{min}^{-1} .100 \mathrm{~cm}^{-2}$ serosa.

\section{EXPERIMENTAL PROCEDURES}

A constant net fluid transport was recorded for 30-60 minutes. Hexamethonium $(10 \mathrm{mg} / \mathrm{kg})$ or atropine $(0.25$ or $0.5 \mathrm{mg} / \mathrm{kg})$ was injected intravenously and fluid transport was followed for another hour. In the lidocaine experiments a $1 \%$ solution of the drug was applied to the serosal surface $(0.5 \mathrm{mg} / \mathrm{cm}$ intestine $)$.

\section{HISTOLOGICAL PROCEDURES}

A $10-15 \mathrm{~mm}$ long part proximal and distal to the experimental segment, as well as part of the experimental segment itself, were cut open and immersion fixed in an isotonic formaldehydeacetic acid solution. ${ }^{10}$ After dehydration in ethanol, embedding in paraffin, and sectioning, the tissue was stained for mast cells in $0.5 \%$ Toluidine blue in $0.5 \mathrm{M} \mathrm{HCl} .1$

DRUGS AND SOLUTIONS

Atropine sulphate, hexamethonium bromide, and naloxone were purchased from Sigma Chemicals, St Louis, MO. The lidocaine solution used is one that is commercially available from Astra AB, Södertälje, Sweden (Xylocain, 1\%). The intestinal mucosa was exposed to a solution with the following composition (mM): $\mathrm{NaCl} 122 ; \mathrm{KCl} 3 \cdot 5 ; \mathrm{KH}_{2} \mathrm{PO}_{4} 1 \cdot 2$; $\mathrm{MgCl}_{2} 1 \cdot 2 ; \mathrm{CaCl}_{2} 2 \cdot 5 ; \mathrm{NaHCO}_{3} 25 ;$ mannitol 30 . The solution administered intra-arterially throughout the experiment contained $110 \mathrm{mM}$ glucose and $40 \mathrm{mM} \mathrm{NaHCO}$.

\section{STATISTICAL ANALYSIS}

The Wilcoxon paired signed rank test or sign test was used when the animals served as their own controls. Otherwise the Mann-Whitney U test was used. Differences resulting in $p$ values of 0.05 or less were considered significant.

\section{Results}

The intestine exhibited obvious macroscopic signs of inflammation (oedema and hyperaemia) on days 6-9 after nematode inoculation. Moreover, many nematode larvae were seen in the intestinal lumen. In histological specimens a significantly reduced number of mucosal mast cells was found. In some experiments the mucosa was completely devoid of mast cells. In the histological sections nematode larvae were also observed. In the macroscopically normal intestine from animals infected 11-14 days before the experiment the number of mast cells was increased while the worms were no longer seen.

Net fluid transport was measured in 26 rats on days 6-9 after they had been infected with the parasites. In nine animals the experiments were performed on days 11-14 after infection. Since rates of fluid transport were similar, regardless of the method used for estimating fluid transport, the results from the two methods were pooled.

Figure 1 and Table I show net fluid transport measured during control conditions lasting for at least 45 minutes in rats injected with the larvae and in those not exposed to the nematode (control rats; Fig 1). Compared with the control animals, both groups of infected rats exhibited a significantly different fluid transport. On days 6-9 fluid transport was lower than in the control rats $(p<0.01)$, and on days $11-14$ it was significantly greater $(\mathrm{p}<0.01)$.

Net fluid transport on days 11-14 after infection was significantly higher than that seen on days $6-9(p<0.0001)$. In eight of 26 animals a net fluid secretion was seen on days 6-9. In three other experiments fluid transport in the jejunum was nil. In all experiments performed on days 11-14 a high rate of net fluid uptake was recorded.

An attempt was made to determine if fluid transport varied between days 6 and 9 . It can be seen in Table $I$ that the average rate of fluid transport reached a minimum value of $6 \mu \mathrm{l} . \mathrm{min}^{-1} .100 \mathrm{~cm}^{-2}$ on day 7 . The variation between animals was so large, however, that no statistically significant difference could be shown between the observed transport rates.

To investigate whether the ENS was involved 


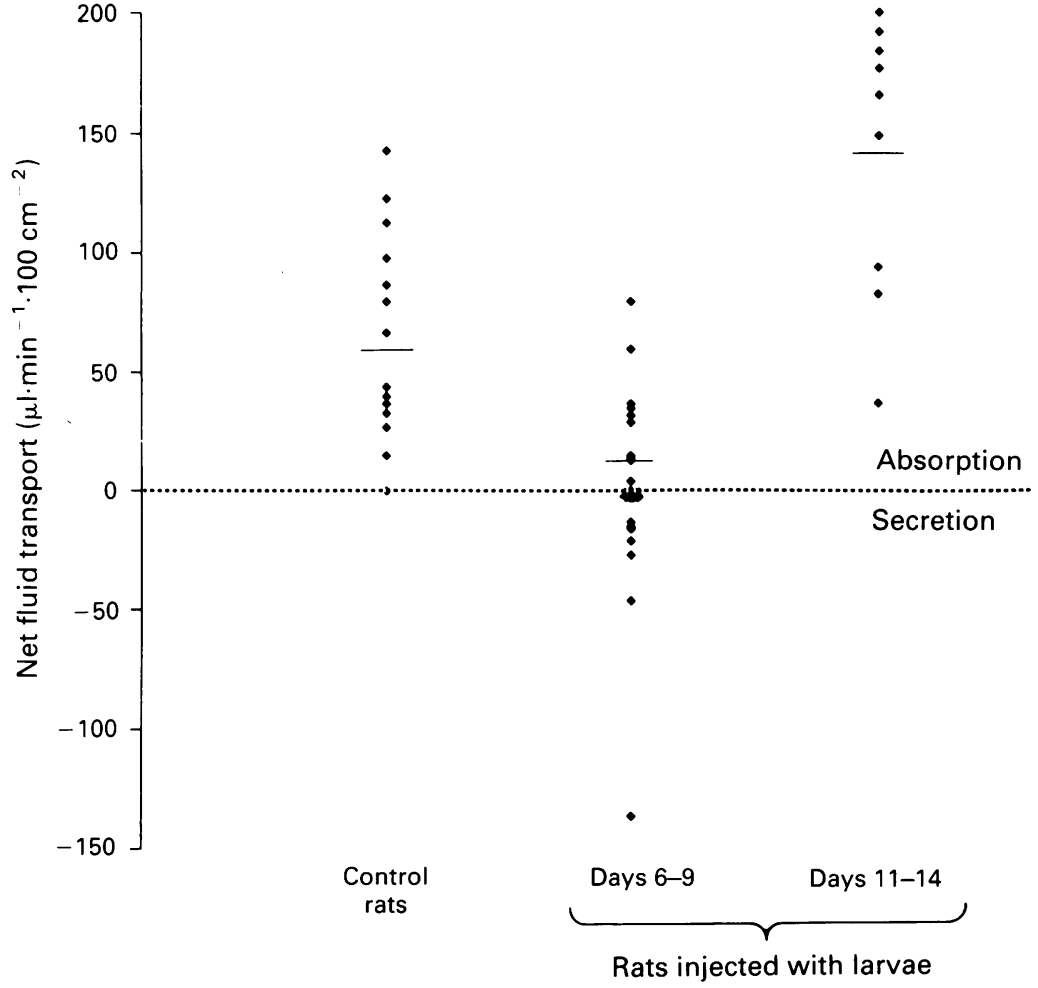

Figure 1: Scattergram of the net fluid transport rates recorded in rats exposed to larvae of Nippostrongylus brasiliensis and in rats not exposed to the parasite (control rats). Observations were made on days 6-9 and 11-14 after giving larvae subcutaneously. Mean values for each group are indicated.

in the secretion seen on days 6-9, two types of experiments were performed. In one series $(\mathrm{n}=9)$ intravenous hexamethonium $(10 \mathrm{mg} / \mathrm{kg})$ was given. This nicotinic receptor blocker significantly influenced fluid transport in the inflamed intestines (Table II, Fig 2) - fluid absorption was increased or fluid secretion was

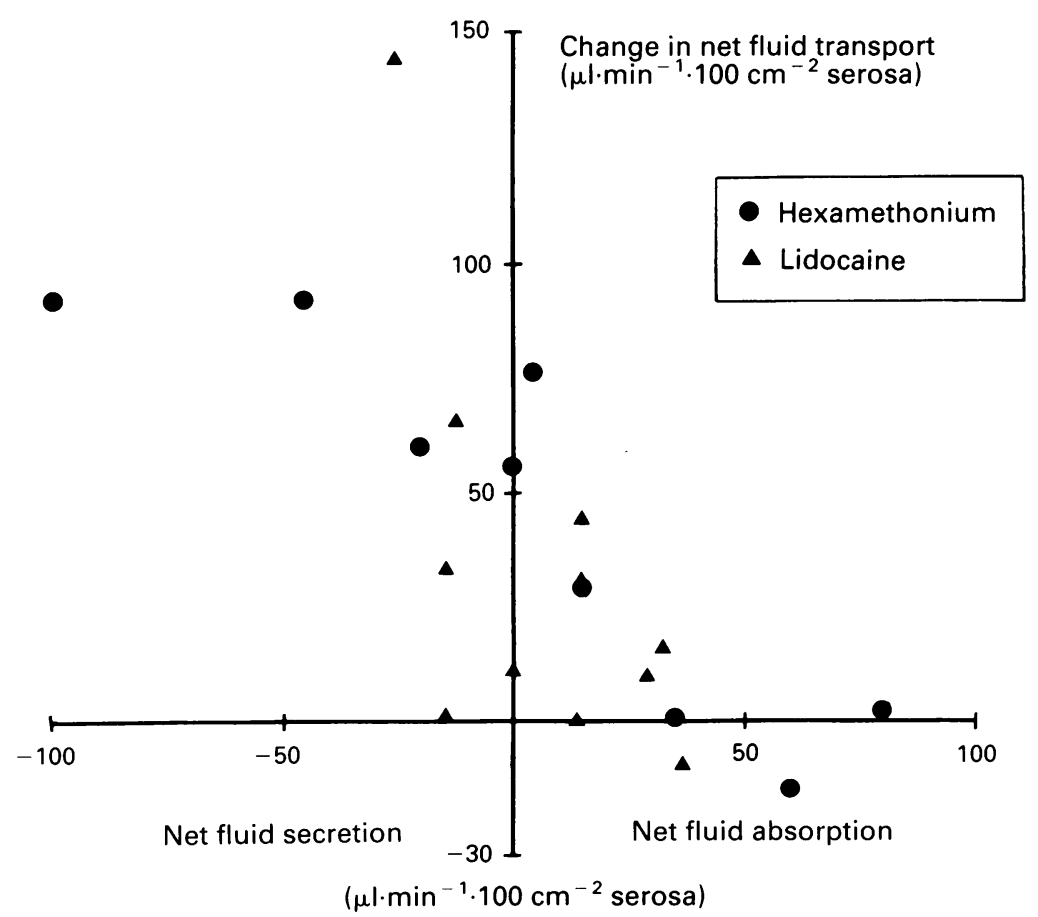

Figure 2: Change in net fluid transport caused by hexamethonium given intravenously or lidocaine placed on the serosal surface plotted versus the rate of net fluid secretion or absorption prevailing before the drug was given. The correlation coefficient was estimated by the method of least squares to be $0 \cdot 72$. The equation relating the two variables was $y=-0 \cdot 78 x+41$.
TABLE I Net fluid absorption $\left(\mu l . \mathrm{min}^{-1} .100 \mathrm{~cm}^{-2}\right)$ in rat jejunum on days 6-9 and $11-14$ after inoculating the animals with larvae of Nippostrongylus brasiliensis

\begin{tabular}{crll}
\hline Day & \multicolumn{2}{c}{ Transport $($ mean $($ SEM $))$} & No of observations \\
\hline 6 & $36(10)$ & & 4 \\
7 & $6(13)$ & $14(9)$ & 8 \\
8 & $15(13)$ & 9 \\
9 & $14(37)$ & & 5 \\
$11-14$ & $142(20)$ & 9 \\
\hline
\end{tabular}

TABLE II The effect of hexamethonium and lidocaine on intestinal net fluid transport $\left(\mu l . \mathrm{min}^{-1} .100 \mathrm{~cm}^{-2}\right)$ in rats injected with the larvae of Nippostrongylus brasiliensis on days 6-9 after injection

\begin{tabular}{|c|c|c|c|}
\hline & \multicolumn{2}{|l|}{ Group } & \multirow[b]{2}{*}{$\begin{array}{l}\text { No of } \\
\text { observations }\end{array}$} \\
\hline & $\begin{array}{l}\text { Control } \\
(\text { mean }(S E M))\end{array}$ & $\begin{array}{l}\text { Drug } \\
(\text { mean }(S E M))\end{array}$ & \\
\hline $\begin{array}{l}\text { Hexmathonium } \\
\text { Lidocaine }\end{array}$ & $\begin{array}{c}-1(22) \\
5(7)\end{array}$ & $\begin{array}{l}43(12)^{\star} \\
34(11)^{\star}\end{array}$ & $\begin{array}{r}9 \\
11\end{array}$ \\
\hline
\end{tabular}

Minus sign denotes net fluid secretion. Asterisk indicates statistically significant difference from control.

significantly decreased in six of nine experiments (Fig 2). A statistically significant increase in fluid uptake was also caused by hexamethonium in experiments on control rats not exposed to the nematode, fluid absorption increasing from 48 (15) to 71 (16) $\mu \mathrm{l} \cdot \mathrm{min}^{-1} .100 \mathrm{~cm}^{-2}$ (mean (SEM); $\mathrm{n}=10$ ).

In another type of experiment performed on days 6-9 after inoculation of the animals with the parasite, the serosal surface of the inflamed segment was exposed to lidocaine ( $1 \%$ solution). Experiments were performed on 11 rats. In eight animals lidocaine decreased fluid secretion or increased fluid absorption (Table II, Fig 2). In control rats lidocaine increased the fluid uptake from 74 (11) to 93 (16) $\mu \mathrm{l} \cdot \mathrm{min}^{-1} .100 \mathrm{~cm}^{-2}$ serosal surface $(n=5)$. This effect was not statistically significant.

The quantitative effects of the drugs were related to the rate of fluid secretion or absorption. The drug was most effective when fluid absorption was low or when the intestine was secreting, as is apparent in Figure 2, in which the change in fluid transport evoked by hexamethonium or lidocaine is plotted versus the rate of net fluid transport recorded before giving the drug. The correlation coefficient between these two variables was $0.72(p<0.01)$.

In four experiments performed on days 6-9 after inoculation, intravenous atropine $(0.25$ or $0.50 \mathrm{mg} \cdot \mathrm{kg}^{-1}$ ) was given. No effect on fluid transport was seen.

A high rate of fluid absorption was recorded on days 11-14. Histological investigation of the intestine did not show any morphological features that could explain the physiological observations.

\section{Discussion}

Larvae of Nippostrongylus brasiliensis injected into the skin migrate to the lungs and move from the third to the fourth stage of their life cycle. Via the trachea and the oesophagus, they eventually reach the small intestine. In the upper half of the jejunum they cause a noticeable inflammatory 
response. Around day 12 the worms are eliminated from the host.

In this report we investigated fluid transport in rats on days 6-9 or 11-14 after having been injected with the larvae. There were obvious macroscopic and microscopic signs of inflammation on days 6-9. A few days later inflammation had diminished and in some animals the jejunum looked macroscopically normal. A noticeable reduction in fluid uptake or an apparent fluid secretion was seen on days 6-9, although the recorded secretion was not as large as one would expect from the rather pronounced inflammatory response in the jejunal segment that was investigated. In contrast, a pronounced net fluid absorption was recorded on days 11-14. These findings agree with results reported by other researchers investigating fluid transport in vivo. Symon studied net fluid and sodium transport in the jejunal segment with the most severe inflammation 10 days after inoculation. ${ }^{12} \mathrm{He}$ found that fluid and electrolyte absorption had become secretion. Similarly, Scofield, studying the entire small intestine, reported decreased fluid absorption on day $10 .{ }^{13}$ Nolla et al made a more thorough analysis, determining fluid transport on days $4,6,9$, and $13 .{ }^{14}$ On days 6 and 9 fluid transport was significantly decreased in the jejunum compared with control values whereas this was not the case on days 13 and 19 after inoculation. Several reports also suggest that the absorption of other nutrients than water, such as hexoses, amino acids, and fatty acids, are decreased in the inflamed jejunum..$^{1416}$

Observations made in vitro on stripped intestinal preparations generally agree with the present findings. Perdue et al measured basic electrical parameters in the rat small intestine in Ussing chambers during experimental conditions similar to those in this study. ${ }^{17}$ The short circuit current (SCC) rose significantly from day 4 to 7 after inoculation, reaching a value twice that of control in the face of an unchanged electrical conductance. Radioactive tracer studies suggested that the increase in the SCC reflected a change in sodium and chloride transport from net absorption to net secretion. On day 21 the monitored electrical parameters were back to control values. Taken together the results from the in vivo and in vitro studies summarised above clearly indicate that the epithelial absorptive capacity of the rat jejunum is appreciably reduced during the days preceding the expulsion of the parasite.

Castro et al used another parasite, Trichinella spiralis, to study the effect of intraluminal parasites on intestinal functions, including fluid and electrolyte transport in vivo. In agreement with our findings, they showed that the rat intestine secreted fluid on day 5 after parasite administration. On day 30 , when the nematodes had been expelled from the gut, fluid transport was back to control values. ${ }^{18}$

The results of this study clearly suggest that the decreased fluid transport seen in the jejunum on days 6-9 after inoculation was at least partly mediated via activation of the enteric nervous system. This conclusion is based on the experimental results obtained with hexamethonium and lidocaine. The relative extent of involvement of the enteric nerves is difficult to ascertain since for technical reasons no control fluid absorption was recorded in the present animals before exposure to the parasite. A rough calculation can, however, be made based on the rate of fluid absorption observed in the control rats. It was about $80 \mu \mathrm{l} . \mathrm{min}^{-1} .100 \mathrm{~cm}^{-2}$ after administration of hexamethonium or lidocaine. From the results reported in Table II and the correlation in Figure 2, it can be calculated that about $50 \%$ of the observed change in fluid transport was mediated via the ENS, when net fluid absorption had stopped. With increasing rates of secretion an increasing fraction of the change in fluid transport caused by the nematode was mediated by nervous mechanisms.

Mucosal mast cells in the rat intestine are drastically affected by an infection with Nippostrongylus brasiliensis. A reduction in number occurs as the larvae infect the intestine. After about a week a dramatic increase in numbers occurs parallel to the elimination of the nematode. ${ }^{19}$ During the initial phase, newly formed mast cell components such as histamine, serotonin, prostaglandins, and leukotrienes may be released. These substances are all known to be mediators of inflammation and are at least partly responsible for the macroscopically obvious inflammation of the intestine on days 6-9. They may also participate in the activation of the enteric nervous system.

Brunsson $^{7}$ studied the effect of an acute inflammatory response in an experimental model using an invasive strain (R5) of Salmonella typhimurium. Net fluid transport was measured 18 hours after inoculating the intestine with the bacterial strain. A net fluid secretion was observed in almost all experiments, secretion averaging $99 \mu \mathrm{l} . \mathrm{min}^{-1} .100 \mathrm{~cm}^{-2}$ serosa in 26 experiments. This secretion is significantly higher than that observed in the present study on days 6-9 (Table I). In Brunssons's experiments, hexamethonium often turned fluid secretion into fluid absorption. Average fluid transport was 48 and -15 (secretion) $\mu 1 . \mathrm{min}^{-1} .100 \mathrm{~cm}^{-2}$ after giving hexamethonium $(n=11)$ and lidocaine $(n=8)$, respectively. These observations suggest that an acute bacterial inflammation evokes a more pronounced intestinal net fluid secretion than the inflammation caused by Nippostrongylus brasiliensis. Using the same value for control absorption as above one can estimate that hexamethonium blocked about $85 \%$ and lidocaine about $50 \%$ of the secretion caused by the bacterium.

To summarise, the observations reported in this study and in that of Brunsson ${ }^{7}$ clearly indicate that ENS plays an important role in the fluid secretion seen in intestinal inflammation. This is not surprising considering that most inflammatory mediators have been shown to be able to stimulate enteric nerves. We showed this in vivo for serotonin ${ }^{8}$ and prostaglandins. ${ }^{20} \mathrm{~A}$ large number of in vitro studies in Ussing chambers have shown that many compounds released by inflammatory cells (eg monokines, various enzymes, and free radicals) activate enteric nerves either directly or via the formation of prostaglandins (for a recent review, see Hinterleiterner and Powell $\left.{ }^{21}\right)$. In fact, the ENS 
can be looked upon as an amplification system for the inflammatory response.

The physiological and pharmacological studies of ENS indicate that there are at least two types of reflexes in this part of the autonomic nervous system. ${ }^{21}$ There exist reflex arches confined to the gastrointestinal wall ('intramural reflexes'). They often contain a cholinergic synapse and can therefore be shown with the use of a nicotinic receptor blocker. Experimental evidence also exists for the presence of axon reflexes in the alimentary canal. Capsaicin is often used as a pharmacological tool to study such a type of reflex. The results of Brunsson's study and the present one indicate that intramural reflex(es) are involved in the fluid secretion caused by intestinal inflammation. To what extent axon reflexes are involved cannot be judged from the results of the present study.

The rate of fluid transport observed on days 11-14 was approximately twice as high as that seen in control animals. A similar observation was made by Scofield ${ }^{10}$ who studied fluid transport in vitro. In a histological investigation of the intestine we were unable to show any morphological changes that could explain the physiological observations. Our earlier studies of fluid transport during various physiological and pathophysiological circumstances suggest that variations of fluid transport are mainly caused by changes in fluid secretion from the crypts. Thus, the observations made on days 11-14 may be explained by a noticeable inhibition of crypt fluid secretion caused by an unknown mechanism.

This work was supported by grants from the Swedish Medica Research Council $(2235,2855)$, the Swedish Society for Medica Sciences, Fredrik and Ingrid Thurings Foundation, Sigurd and Elsa Goljes Foundation and the Medical School at the University of Göteborg.

1 Cassuto J, Jodal $M$, Tuttle R, Lundgren $\mathrm{O}$. On the role of intramural nerves in the pathogenesis of cholera toxininduced intestinal secretion. Scand $\mathcal{F}$ Gastroent 1981; 16: 377-84

2 Cassuto J, Fahrenkrug J, Jodal M, Tuttle R, Lundgren O. Release of vascactive intestinal polypeptide from the cat small intestine exposed to cholera toxin. Gut 1981; 22: 95863.

3 Cassuto J, Jodal M, Lundgren $\mathrm{O}$. The effect of nicotinic and muscarinic receptor blockade on cholera toxin induced intestinal secretion in rats and cats. Acta Physiol Scand 1982; intestinal secre $573-7$.

4 Cassuto J, Siewerth A, Jodal M, Lundgren O. The involvement of intramural nerves in cholera toxin induced intestinal ment of intramural nerves in cholera toxin induced in

5 Lundgren O, Svanvik J, Jivegård L. Enteric nervous system. I. Physiology and pathophysiology of the intestinal tract. Dig Dis Sci 1989; 34: 264-83.

6 Jodal M. Neuronal influence on intestinal transport. 7 Intern Med 1990; 228 (supple 732): 125-32.

7 Brunsson I. Enteric nerves mediate the fluid secretory response due to Salmonella typhimurium $\mathrm{R} 5$ infection in the rat small intestine. Acta Physiol Scand 1987; 131: 609-17.

8 Cassuto J, Jodal $M$, Tuttle R, Lundgren $O$. 5-hydroxytryptamine and cholera secretion. Scand F Gastroenterol 1982; 17: 695-703.

9 Jodal M, Hallbäck D-A, Svanvik J, Lundgren O. A method for the continous study of net water transport in the feline small bowel. Acta Physiol Scand 1975; 95: 441-7.

10 Enerbäck L. Mast cells in rat gastrointestinal mucosa. I. Effect of fixation. Acta Pathologica et Microbiologica Scandinavica 1966; 66: 289-302.

11 Enerbäck L. Mast cells in rat gastrointestinal mucosa. II. Dye binding and metachromatic properties. Acta Pathologica et Microbiologica Scandinavica 1966; 66: 303-12.

12 Symons LEA. Pathology of infestation of the rat with nippostrongylus muris (Yokogawa). III. Jejunal fluxes in vivo of water, sodium, and chloride. Austr $\mathcal{F}$ Biol Sci 1960; 13: $171-9$.

13 Scofield AM. Intestinal absorption of hexoses in rats infected with nippostrongylus brasiliensis. Int $\mathcal{F}$ Parasitol 1977; 7: with nippc

14 Nolla H, Bristol JR, Mayberry LF. Nippostrongylus brasiliensis: malabsorption in experimentally infected rats. brasiliensis: malabsorption in

15 Symons LEA. Pathology of infestation of the rat with nippostrongylus muris (Yokogawa). IV. The absorption of glucose and histidine. Austr $\mathcal{F}$ Biol Sci 1960; 13: 180-7.

16 Symons LEA, Gibbins JR, Jones WO. Jejunal malabsorption in the rat infected by the nematode nippostrongylus brasiliensis. Int $\mathcal{F}$ Parasitol 1971; 1: 179-87.

17 Perdue MH, Marshall J, Masson S. Ion transport abnormalities in inflamed rat jejunum. Involvement of mast cells and nerves. Gastroenterology 1990; 98: 561-7.

18 Castro GA, Hessel JJ, Whalen G. Altered intestinal fluid movement in response to Trichinella spiralis in immunized movement in response to Trichinella spir.
rats. Parasite Immunol 1979; 1: 259-66.

19 Allenmark S, Dahlström A. Amines of the mucosal mast cells of the gut in normal and nematode infected rats. cells of the gut in normal and

20 Brunsson I, Sjöqvist A, Jodal M, Lundgren O. Mechanisms underlying the small intestinal fluid secretion caused by arachidonic acid, prostaglandin $\mathrm{E}_{1}$ and prostaglandin $\mathrm{E}_{2}$ in the rat in vivo. Acta Physiol Scand 1987; 130: 633-42.

21 Hinterleitner ThA, Powell DW. Immune system control of intestinal ion transport. Proc Soc Exp Biol Med 1991; 197 249-60.

22 Lundgren $O$. Neuroimmune modulation of epithelia function. An overview, Ann NY Acad Sci 1992; 667: 305-27.

23 Eklund S, Sjöqvist A, Fahrenkrug J, Jodal M, Lundgren $O$. Somatostation and methionine-enkephalin inhibit cholera vasoactive intestinal polypeptide in the cat in vivo. Acta Physiol Scand 1988; 133: 551-7. 